University of Wollongong

Research Online

Australian Institute for Innovative Materials -

Papers

Australian Institute for Innovative Materials

$1-1-2012$

Optical parameters of ZnTe determined using continuous-wave terahertz radiation

E Constable

University of Wollongong, evanc@uow.edu.au

Roger A. Lewis

University of Wollongong, roger@uow.edu.au

Follow this and additional works at: https://ro.uow.edu.au/aiimpapers

Part of the Engineering Commons, and the Physical Sciences and Mathematics Commons

Research Online is the open access institutional repository for the University of Wollongong. For further information contact the UOW Library: research-pubs@uow.edu.au 


\title{
Optical parameters of ZnTe determined using continuous-wave terahertz radiation
}

\begin{abstract}
The optical parameters of three ZnTe crystal wafers of different thicknesses were determined using transmittance measurements of continuous-wave terahertz radiation from a two-color photomixing source. The parameters are extracted by fitting the transmittance data with theoretical curves generated using a Drude-Lorentz dielectric model of the crystal and a bootstrap statistical analysis of the fits. It was found at room temperature that the low and high frequency dielectric constants are $\epsilon(0)=9.8 \pm 0.2$ and $\epsilon(\infty)=7.3 \pm 0.6$, respectively. The transverse optical phonon frequency was found to be $V T O=6.0 \pm 1.3$ $\mathrm{THz}$. Sample specific properties such as the plasma, collision, and phonon damping frequencies were determined and used for an approximate calculation of carrier concentration. The results are compared with a comprehensive review of earlier values from the literature. Our results are consistent with previous work, falling within the spread of accepted values, and demonstrate that this method is particularly suited for determining the low and high frequency dielectric constants of semiconductor samples.
\end{abstract}

\section{Keywords}

znte, optical, determined, parameters, continuous, wave, terahertz, radiation

\section{Disciplines}

Engineering | Physical Sciences and Mathematics

\section{Publication Details}

Constable, E \& Lewis, RA (2012), Optical parameters of ZnTe determined using continuous-wave terahertz radiation, Journal of Applied Physics, 112(6), pp. 063104-1-063104-6. 


\section{AIP Applied Physics}

\section{Optical parameters of ZnTe determined using continuous-wave terahertz radiation}

E. Constable and R. A. Lewis

Citation: J. Appl. Phys. 112, 063104 (2012); doi: 10.1063/1.4752405

View online: http://dx.doi.org/10.1063/1.4752405

View Table of Contents: http://jap.aip.org/resource/1/JAPIAU/v112/i6

Published by the American Institute of Physics.

\section{Related Articles}

Temperature dependent graphene suspension due to thermal Casimir interaction Appl. Phys. Lett. 101, 113118 (2012)

Low-energy electron transmission imaging of clusters on free-standing graphene Appl. Phys. Lett. 101, 113117 (2012)

Structural and optoelectronic properties of P3HT-graphene composites prepared by in situ oxidative polymerization

J. Appl. Phys. 112, 054327 (2012)

Electronic structure and optical properties of $\beta$-FeSi2(100)/Si(001) interface at high pressure Appl. Phys. Lett. 101, 111909 (2012)

Solid films and transports in cellular foams

J. Appl. Phys. 112, 054911 (2012)

\section{Additional information on J. Appl. Phys.}

Journal Homepage: http://jap.aip.org/

Journal Information: http://jap.aip.org/about/about_the_journal

Top downloads: http://jap.aip.org/features/most_downloaded

Information for Authors: http://jap.aip.org/authors

\section{ADVERTISEMENT}

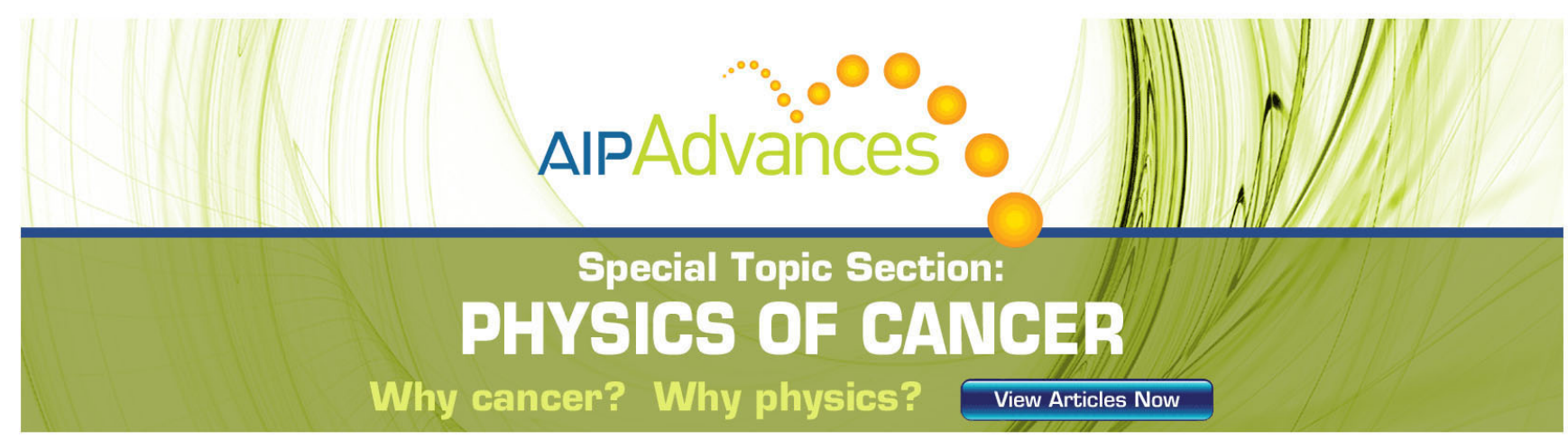




\title{
Optical parameters of ZnTe determined using continuous-wave terahertz radiation
}

\author{
E. Constable ${ }^{\text {a) }}$ and R. A. Lewis ${ }^{\text {b) }}$ \\ Institute for Superconducting and Electronic Materials, University of Wollongong, \\ Wollongong NSW 2522, Australia
}

(Received 1 May 2012; accepted 15 August 2012; published online 18 September 2012)

\begin{abstract}
The optical parameters of three ZnTe crystal wafers of different thicknesses were determined using transmittance measurements of continuous-wave terahertz radiation from a two-color photomixing source. The parameters are extracted by fitting the transmittance data with theoretical curves generated using a Drude-Lorentz dielectric model of the crystal and a bootstrap statistical analysis of the fits. It was found at room temperature that the low and high frequency dielectric constants are $\epsilon(0)=9.8 \pm 0.2$ and $\epsilon(\infty)=7.3 \pm 0.6$, respectively. The transverse optical phonon frequency was found to be $\nu_{\mathrm{TO}}=6.0 \pm 1.3 \mathrm{THz}$. Sample specific properties such as the plasma, collision, and phonon damping frequencies were determined and used for an approximate calculation of carrier concentration. The results are compared with a comprehensive review of earlier values from the literature. Our results are consistent with previous work, falling within the spread of accepted values, and demonstrate that this method is particularly suited for determining the low and high frequency dielectric constants of semiconductor samples. (C) 2012 American Institute of Physics. [http://dx.doi.org/10.1063/1.4752405]
\end{abstract}

\section{INTRODUCTION}

The rapid development of optoelectronics for use in terahertz $(\mathrm{THz})$ emission and detection in recent years has led to the development of new technologies based on a variety of different materials. ${ }^{1}$ Of particular interest is the II-VI polar semiconductor zinc telluride (ZnTe), which exhibits birefringence when illuminated with $\mathrm{THz}$ radiation. ${ }^{2,3}$ The magnitude of the birefringence is dependent on the incident $\mathrm{THz}$ power, which in turn can be monitored using optical sampling, hence forming a $\mathrm{THz}$ detector system. ${ }^{4}$ Conversely a high-powered optical pulse incident on a ZnTe crystal will experience nonlinear effects, which can produce $\mathrm{THz}$ radiation. $^{2-5}$ For device applications such as this, it is important that accurate values of the optical parameters are known. While the various properties of $\mathrm{ZnTe}$ have been investigated for some time, ${ }^{6-11}$ there is still considerable discrepancy between the results in the literature. ${ }^{12-23}$

In this article we present new measurements for the optical parameters of ZnTe by performing transmittance measurements with theoretical fits in the $\mathrm{THz}$ range. $\mathrm{THz}$ radiation is particularly useful for characterizing semiconductor materials due to its strong coupling with the free electrons and phonon interactions of the material. ${ }^{22,24}$ To perform these measurements we use a two-color photomixing $\mathrm{THz}$ source as a tunable continuous-wave $\mathrm{THz}$ spectrometer to measure the transmittance at normal incidence of three ZnTe crystal wafers with different thicknesses. By fitting a theoretical transmittance curve to the experimental data using a DrudeLorentz model of the dielectric function and performing a statistical bootstrap analysis of the fits, the various optical parameters are determined. We present this information in

\footnotetext{
${ }^{\text {a)} E l e c t r o n i c ~ m a i l: ~ e c 028 @ u o w m a i l . e d u . a u . ~}$

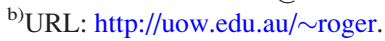

summary with results given in the literature in order to provide a reference for the parameters of $\mathrm{ZnTe}$.

In contrast to traditional electrical transport measurements, ${ }^{6}$ this method does not require the attachment of electrodes, potentially damaging the sample. Previous optical methods for characterizing ZnTe have had success using far infrared interference and grating spectrometers and applying Drude dispersion theory or the Kramers-Kronig dispersion relation. ${ }^{14}$ However, these measurements often require either thin film samples for transmission measurements or complicated optical setups for reflection measurements. ${ }^{13,14,22} \mathrm{THz}$ time-domain spectroscopy measurements performed more recently have had success using transmission measurements due the low absorption of $\mathrm{THz}$ frequencies by ZnTe crystals. ${ }^{20,21}$ However such setups have large startup costs due to the requirement of femtosecond lasers for their operation. As photomixing technologies continue to be improved, through development of new materials able to photomix efficiently using telecommunication wavelengths, ${ }^{25}$ such two-color systems will prove to be more widely accessible.

\section{THEORY}

Starting with Maxwell's equations and treating the sample as an insulator, the dispersion relation inside the crystal for a single mode in one dimension can be represented as a function of the angular frequency of the incident $\mathrm{THz}$ radiation $(\omega=2 \pi \nu),{ }^{26}$

$$
k^{2}=\frac{\omega^{2}}{c^{2}} \epsilon(\omega),
$$

where both the wave number, $k$, and the angular frequencydependent dielectric function, $\epsilon(\omega)$, are complex and $c$ is the speed of light. In solving Maxwell's equations, when considering the crystal polarization and current density, it is found 
that for a polar material containing free charge carriers such as $\mathrm{ZnTe}$ the dielectric function can be modelled using three terms taking the form, ${ }^{22}$

$$
\epsilon(\omega)=\epsilon(\infty)-\frac{\epsilon(\infty) \omega_{\mathrm{p}}^{2}}{\omega^{2}+i \omega \gamma}+\frac{(\epsilon(0)-\epsilon(\infty)) \omega_{\mathrm{TO}}^{2}}{\left(\omega_{\mathrm{TO}}^{2}-\omega^{2}\right)+i \omega \Gamma},
$$

where $\epsilon(\infty)$ is the high frequency dielectric constant, also referred to as the optical, lattice, or ac dielectric constant, $\epsilon(0)$ is the low frequency dielectic constant and is also known as the static or dc dielectric constant, $\omega_{\mathrm{p}}$ is the angular plasma frequency $\left(\omega_{\mathrm{p}}^{2}=n e^{2} / m^{*} \epsilon_{0} \epsilon(\infty)\right)$, $\omega_{\text {TO }}$ is the angular transverse optical phonon frequency, $\gamma$ is the plasma damping constant or collision frequency, and $\Gamma$ is the phonon damping constant. The first term we call the atomic term as it results from the displacement of the atomic electrons in relation to their effectively stationary ions in the lattice. The second term comes from the Drude model assumption of the motion of free electrons with respect to the lattice. The final term, known as the Lorenz term, represents the oscillation of the positive $\mathrm{Zn}$ ions relative the negatively charged neighboring Te ions.

It is convenient, when working with a complex dielectric function, to separate the real and imaginary parts by defining

$$
\epsilon \equiv \epsilon_{1}+i \epsilon_{2}
$$

where

$$
\epsilon_{1}=\epsilon(\infty)-\frac{\epsilon(\infty) \omega_{\mathrm{p}}^{2}}{\omega^{2}+\gamma^{2}}+\frac{(\epsilon(0)-\epsilon(\infty))\left(\omega_{\mathrm{TO}}^{2}-\omega^{2}\right) \omega_{\mathrm{TO}}^{2}}{\left(\omega_{\mathrm{TO}}^{2}-\omega^{2}\right)^{2}+\omega^{2} \Gamma^{2}},
$$

and

$$
\epsilon_{2}=\frac{\epsilon(\infty) \omega_{\mathrm{p}}^{2} \gamma}{\omega^{3}+\omega \gamma^{2}}-\frac{(\epsilon(0)-\epsilon(\infty)) \omega \omega_{\mathrm{TO}}^{2} \Gamma}{\left(\omega_{\mathrm{TO}}^{2}-\omega^{2}\right)^{2}+\omega^{2} \Gamma^{2}}
$$

We give the same treatment to the complex wave number, defining

$$
k \equiv \alpha+i \beta=\frac{\omega}{c} N=\frac{\omega}{c}(\eta+i \kappa) .
$$

Here, $N$ is the complex index of refraction, $\alpha$ determines the wavelength $(\lambda \equiv 2 \pi / \alpha)$, and $\beta$ determines the attenuation length $(\zeta \equiv 1 / \beta)$. Relating this to the dispersion relation (Eq. (1)) gives

$$
\alpha=\frac{\omega}{c} \eta=\frac{\omega}{c}\left(\frac{|\epsilon|+\epsilon_{1}}{2}\right)^{\frac{1}{2}}
$$

and

$$
\beta=\frac{\omega}{c} \kappa=\frac{\omega}{c}\left(\frac{|\epsilon|-\epsilon_{1}}{2}\right)^{\frac{1}{2}}
$$

where $|\epsilon| \equiv\left(\epsilon_{1}^{2}+\epsilon_{2}^{2}\right)^{\frac{1}{2}}$.
The propagation of linear polarized $\mathrm{THz}$ radiation through the ZnTe samples at normal incidence is described by standard Fabry-Perot theory. The theoretical transmissivity is calculated by summing the multiple internal reflections from each face of the sample wafer, which are themselves described by the Fresnel equations. In doing so we find that the transmissivity is given by

$$
\tau=\frac{\left(1-r^{2}\right) e^{i \delta}}{1-r^{2} E_{0} e^{2 i \delta}}
$$

where $r=|r| e^{i \phi_{r}}$ is complex and given by the Fresnel equations for an infinite half-space at normal incidence. $\delta=k d$ $=(\alpha+i \beta) d$ represents the phase difference and absorption between successive internal reflections, with $d$ the sample thickness. The magnitude and phase of the infinite half space reflection coefficient are given by ${ }^{26}$

$$
|r|=\left[\frac{(1-\eta)^{2}+\kappa^{2}}{(1+\eta)^{2}+\kappa^{2}}\right]^{\frac{1}{2}}
$$

and

$$
\phi_{r}=\arctan \left[\frac{2 \kappa}{1-\eta^{2}-\kappa^{2}}\right] .
$$

To find the observable transmittance we take $T=\tau \tau^{*}$, where $T=\frac{I_{t}}{I_{0}}$ is the ratio of the transmitted radiation with the incident radiation.

$$
\begin{aligned}
\tau \tau^{*} & =\frac{\left(1-|r|^{2} e^{2 i \phi_{r}}\right) e^{i \alpha d} e^{-\beta d}}{1-|r|^{2} e^{2 i \phi_{r}} e^{2 i \alpha d} e^{-2 \beta d}} \frac{\left(1-|r|^{2} e^{-2 i \phi_{r}}\right) e^{-i \alpha d} e^{-\beta d}}{1-|r|^{2} e^{-2 i \phi_{r}} e^{-2 i \alpha d} e^{-2 \beta d}} \\
& =\frac{e^{-2 \beta d}\left(1-|r|^{2} e^{2 i \phi_{r}}\right)\left(1-|r|^{2} e^{-2 i \phi_{r}}\right)}{\left(1-|r|^{2} e^{2 i\left(\phi_{r}+\alpha d\right)} e^{-2 \beta d}\right)\left(1-|r|^{2} e^{-2 i\left(\phi_{r}+\alpha d\right)} e^{-2 \beta d}\right)} .
\end{aligned}
$$

Expanding and simplifying through both the numerator and denominator gives

$$
T=\frac{e^{-2 \beta d}\left(1-|r|^{2}\right)^{2}+4|r|^{2} \sin ^{2}\left(\phi_{r}\right)}{\left(1-|r|^{2} e^{-2 \beta d}\right)^{2}+4|r|^{2} e^{-2 \beta d} \sin ^{2}\left(\alpha d+\phi_{r}\right)} .
$$

Substituting back through Eqs. (4), (5), (7), (8), (10), and (11) gives $T$ as a function of $\omega, \epsilon(0), \epsilon(\infty), \omega_{\mathrm{p}}, \omega_{\mathrm{TO}}, \gamma, \Gamma$, and $d$.

\section{METHOD}

\section{A. Experiment}

The transmittance measurements are performed using a frequency tunable, continuous-wave $\mathrm{THz}$ source known as a two-color, optical-heterodyne, or photomixer system. ${ }^{27}$ The system (Fig. 1) operates by pumping a gallium-arsenide/lowtemperature-grown gallium-arsenide (GaAs/LTG GaAs) heterostructure fabricated with interdigitated electrodes and a log-periodic antenna with two frequency-offset near-infrared (NIR) diode lasers (Toptica DL-100, $\lambda_{0}=853 \pm 3 \mathrm{~nm}$ ). Photocarriers are excited in the LTG GaAs layer by the NIR 


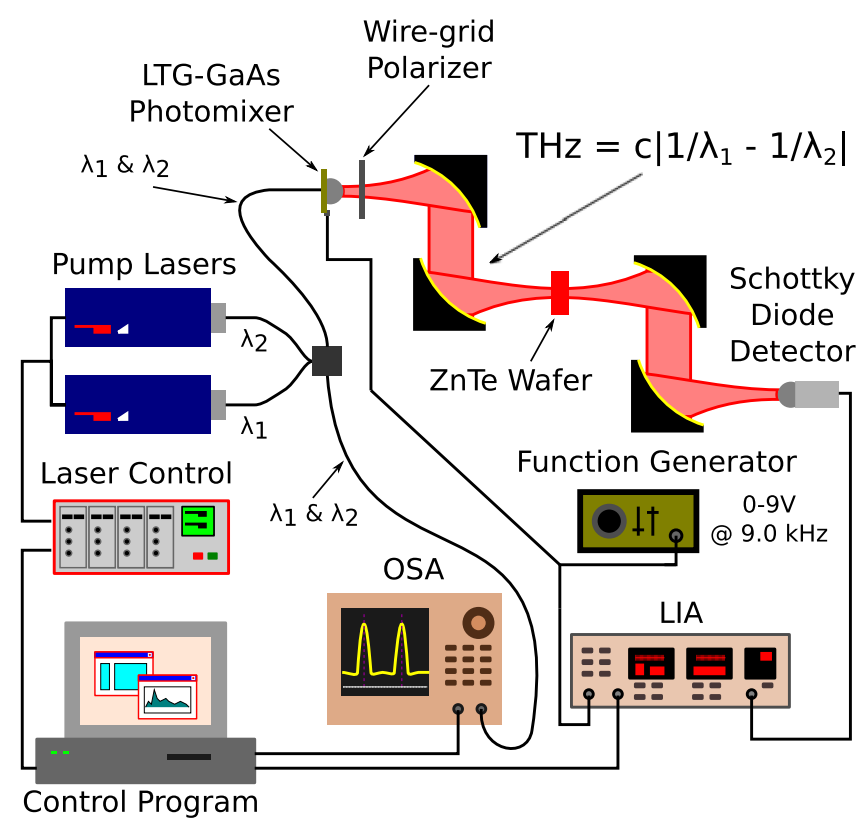

FIG. 1. Two-color THz spectrometer setup for measuring semiconductor transmittance.

radiation and form Hertzian dipoles under the bias of the electrodes. LTG GaAs has the ideal properties of high carrier mobility and fast recombination times that allow the beat frequency of the mixed pumping beams to modulate the amplitude of the Hertzian dipoles, causing the photomixer to radiate a $\mathrm{THz}$ signal equal to the difference frequency of the two pump lasers. A hyper-hemispherical silicon lens fixed to the photomixer initially collimates the $\mathrm{THz}$ beam. The emitted radiation is then sent through a set of gold-plated off-axis parabolic mirrors that further collimate, focus to a sample, then re-collimate, and finally focus onto a detector in the manner seen in Fig. 1. The detector consists of a room temperature operated Schottky diode detector manufactured by advanced compound semiconductor technologies (ACST) at the Technical University of Darmstadt. ${ }^{28}$ The THz signal is electronically chopped by modulating the photomixer bias, using a function generator which also acts as a reference for a lock in amplifier (LIA) that monitors the signal. The two diode lasers are frequency tunable, and a spectrum of $\mathrm{THz}$ frequencies is produced by tuning the frequency difference of the two lasers. The frequency of the THz signal is measured at regular intervals using an optical spectrum analyser (OSA) to determine the frequency of each of the diode lasers and hence their difference. The ZnTe wafers are placed in the beam path of the $\mathrm{THz}$ radiation, and a spectrum is taken by measuring the transmitted intensity as a function of frequency from 0.15 to $1.0 \mathrm{THz}$ at a resolution of $0.001 \mathrm{THz}$. A wire-grid linear polarizer is also placed in the beam path before the sample to ensure polarization of the incident radiation. The ratio of the sample spectrum with a reference spectrum gives the experimental transmittance.

\section{B. Analysis}

For the purposes of analysis the thickness of each wafer was measured separately and taken as a known constant. Our theoretical model therefore consists of six variable parameters $\epsilon(0), \epsilon(\infty), \omega_{\mathrm{TO}}, \Gamma, \omega_{\mathrm{p}}$, and $\gamma$. The transmittance is nonlinearly dependent on these parameters. The theoretical transmittance is fitted to the experimental data using a MarquardtLevenberg nonlinear least-squares fitting algorithm implemented by a standard graphing application (WaveMetrics: Igor Pro). Unlike a fitting function with linear dependence on its variable parameters, there do not exist exact solutions for the optimal parameters and their respective uncertainties for fitting functions with nonlinear parameter dependence. ${ }^{29} \mathrm{Mul}-$ tiple minima for the variance-of-fit will often exist, making the fit, and therefore the resulting parameter values, sensitive to the initial values used for the fitting algorithm. ${ }^{29}$ Furthermore, the uncertainties generated by the covariance matrix are linear approximations and are underestimated as they neglect the cross-correlation between each parameter. ${ }^{30}$ In order to obtain accurate parameter values with genuine uncertainties, we implement a multiple-step fitting process utilizing the established bootstrap method. ${ }^{29-31}$

The first step involves a Marquardt-Levenberg nonlinear least-squares fit of the experimental data, with all but one of the fitting parameters fixed using initial conditions gathered from the literature. Once the varied parameter has been adjusted to its best fit, it is then fixed and a subsequent parameter is varied. Results that produce nonphysical values are rejected, and the process is repeated after adjusting the initial conditions. After this process the same fitting procedure is performed with all of the parameters freely variable. The results of this fit are then used in a bootstrap procedure to determine the optimal parameter values and uncertainties.

The bootstrap procedure involves generating a large number of synthetic data tables using the theoretical fitting function and parameter values produced by the last fitting algorithm. An effective experimental noise is applied to each of the synthetic data tables by adding values randomly sampled with replacement from the residuals table that is a result of the difference between the experimental data and the Marquardt-Levenberg fit. The Marquardt-Levenberg nonlinear least-squares fitting algorithm is then applied to each of the synthetic data tables producing a new set of optimal parameter values for each table. The parameter values for each fit are stored in their own tables, generating a population of possible values for each parameter. The optimal parameter values are then determined by statistical analysis of the parameter populations.

To be sure the optimal parameter values are reliable, the cross-correlation coefficients for each parameter pair are also calculated for every bootstrap fit. Statistical analysis of the cross-correlation coefficient populations allows us to monitor any cross-correlations between the parameters that would affect the accuracy of the results. As a rule of thumb, parameters with high cross-correlation will have crosscorrelation coefficients with magnitudes beyond \pm 0.95 with \pm 1.0 representing complete correlation. ${ }^{29,30} \mathrm{We}$ also monitor cross-correlations by observing scatter graphs produced by plotting the bootstrap determined parameter values against each other. Cross-correlated parameter pairs will produce a dependent curve when plotted against each other. Uncorrelated parameter pairs will have circular scatter graphs with Gaussian cross-sections. ${ }^{30}$ 


\section{RESULTS AND DISCUSSION}

In this section the experimental measurements are presented along with the theoretical fits produced using the optimal parameter values obtain through the bootstrap analysis. The results of the analysis are then tabulated with a comprehensive list of other results taken from the literature. While our new values are consistent with the previously published values, the table demonstrates the spread in the accepted values and highlights the need for accurate results. Finally, a discussion on each variable is given, with consideration given to the previously published values.

\section{A. Measurements}

The spectrum of a $0.5 \mathrm{~mm} \mathrm{ZnTe}$ wafer is shown against a reference spectrum in Fig. 2. The characteristic water vapor absorption lines are observed at 0.55 and $0.75 \mathrm{THz}$. The interference fringes (or Fabry-Perot oscillations) due to internal reflections at the sample/air interfaces evident in the sample spectrum are easily resolved by the spectrometer. Even without detailed analysis, the relatively high resistivity of the sample can be seen in the spectrum. The maxima in the fringes is approximately equal to the reference spectrum, indicating low absorption. The ratio of the sample spectrum with the reference spectrum gives the transmittance, shown in Fig. 3 for $0.3,0.5$, and $1.0 \mathrm{~mm}$ wafer thicknesses. The FabryPerot oscillations now dominate the transmittance data and are used to aid the theoretical fitting. The separation of these fringes is approximately $0.16,0.10$, and $0.05 \mathrm{THz}$ for the 0.3 , 0.5 , and $1.0 \mathrm{~mm}$ samples, respectively. Outlying data points in the transmittance plots are attributed to random noise in the measurements. Points where the transmittance is

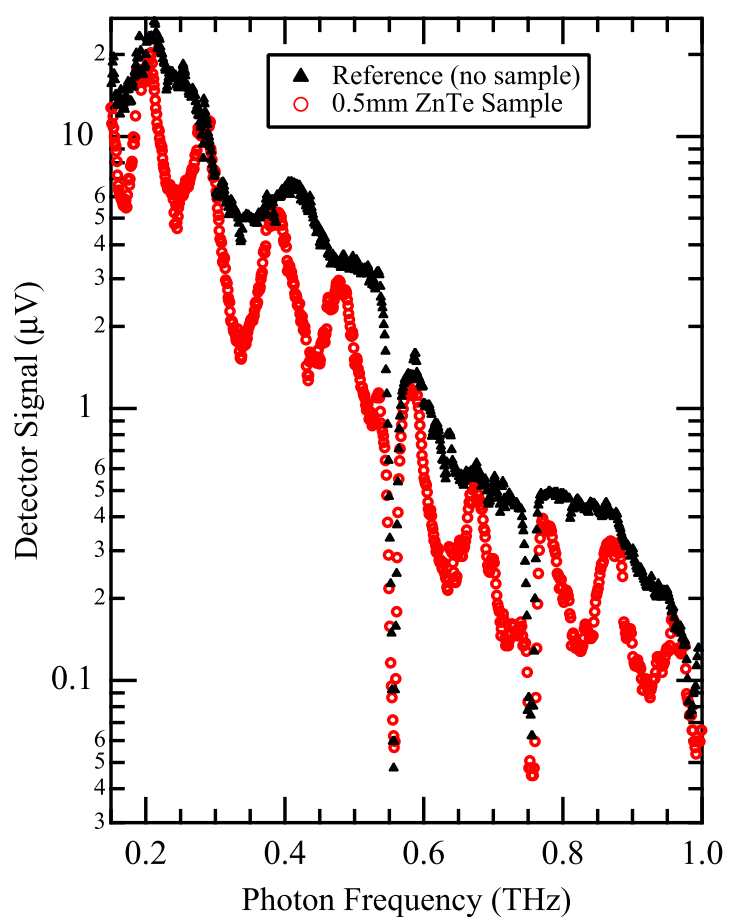

FIG. 2. Spectrum of $0.5 \mathrm{~mm}$ thick ZnTe wafer (circles) with reference (solid triangles).

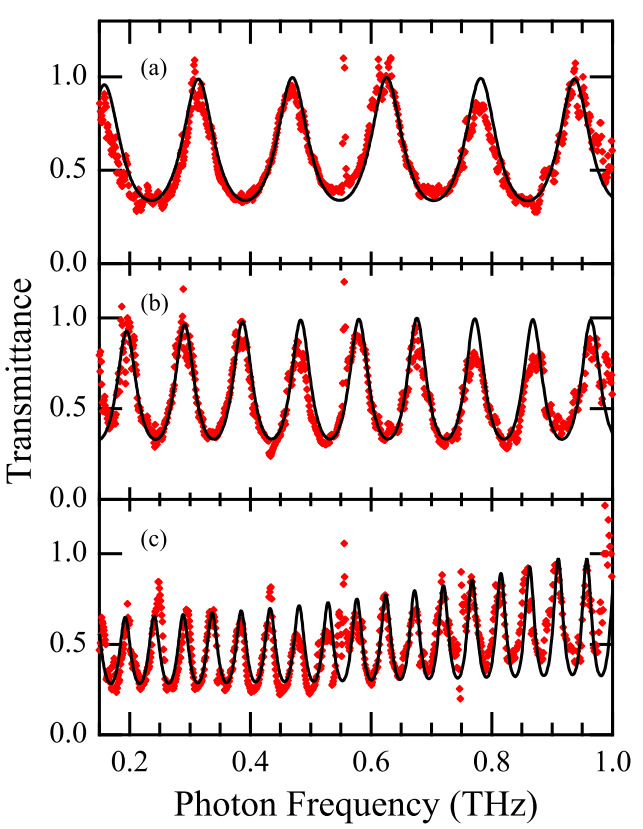

FIG. 3. Transmittance measurements for three ZnTe wafers (solid diamonds) with thicknesses, $d$, of $0.3 \mathrm{~mm}$ (a), $0.5 \mathrm{~mm}$ (b), and $1.0 \mathrm{~mm}$ (c) fitted with theoretical curve (solid curve).

greater than 1.0 occur when the sample measurements have experienced noise giving a higher signal relative to the reference measurement. In particular, there are consistent outliers at the $0.55 \mathrm{THz}$ water absorption peak, when both the reference and sample spectra exhibit a low signal to noise. When performing the fitting algorithm these points are masked to assist in a better fit.

\section{B. Dielectric parameters}

The results of the bootstrap analysis used to plot the theoretical curves in Fig. 3 are given in Table I. They represent the rounded average parameter values taken over 1000 bootstrap samples with the uncertainty given as the standard deviation rounded to the nearest significant figure stated for the parameter value. We have converted the angular frequencies $(\omega)$ stated in Sec. II to usual frequencies $(\nu)$ which are more often reported. Since the plasma frequency $\left(\nu_{\mathrm{p}}\right)$, collision frequency $(\gamma)$, and phonon damping $(\Gamma)$ parameters all depend on an individual sample's doping and crystal imperfections, they are often not reported and thus have not been included in many of the previous results. They have been left in this analysis to demonstrate the capability of this technique. A more detailed discussion of the results for each parameter will follow.

\section{Low and high frequency dielectric constants}

Both the low and high frequency dielectric constants are intrinsic optical properties of the crystal. It is for these parameters where there is the biggest variance in previously published values. Due to the low frequency, relative to the phonon frequency, at which these measurements are performed, we expect accurate results from the low frequency dielectric constant. This is evident in how the results 
TABLE I. Optical parameters for ZnTe at room temperature determined by a statistical bootstrap analysis of the theoretical fits in Fig. 3. The results are compared with previous results from literature $\left(\nu_{\mathrm{p}}=\omega_{\mathrm{p}} / 2 \pi, \nu_{\mathrm{TO}}=\omega_{\mathrm{TO}} / 2 \pi\right)$.

\begin{tabular}{|c|c|c|c|c|c|c|}
\hline & $\epsilon(0)$ & $\epsilon(\infty)$ & $\nu_{\mathrm{TO}}(\mathrm{THz})$ & $\Gamma(\mathrm{THz})$ & $\nu_{\mathrm{p}}(\mathrm{THz})$ & $\gamma(\mathrm{THz})$ \\
\hline This work $(0.3 \mathrm{~mm})$ & $9.7 \pm 0.1$ & $7.3 \pm 0.6$ & $6.4 \pm 0.8$ & $0.4 \pm 0.1$ & $0.03 \pm 0.01$ & $0.5 \pm 0.1$ \\
\hline This work $(0.5 \mathrm{~mm})$ & $9.9 \pm 0.1$ & $7.4 \pm 0.2$ & $6.8 \pm 0.2$ & $0.3 \pm 0.1$ & $0.03 \pm 0.01$ & $0.5 \pm 0.1$ \\
\hline This work $(1.0 \mathrm{~mm})$ & $9.9 \pm 0.1$ & $7.1 \pm 0.4$ & $5.0 \pm 0.3$ & $2.0 \pm 0.1$ & $0.1 \pm 0.1$ & $11.7 \pm 0.7$ \\
\hline Aven $^{6}$ & 10.1 & 8.26 & - & - & - & - \\
\hline Berlincourt $^{7}$ & 10.1 & - & - & - & - & - \\
\hline Marple $^{9}$ & - & 7.28 & - & - & - & - \\
\hline Manabe $^{10}$ & 9.1 & 6.7 & 5.37 & 0.017 & - & - \\
\hline Mitra $^{11}$ & - & - & 5.37 & - & - & - \\
\hline Rode $^{12}$ & 9.67 & 7.28 & - & - & - & - \\
\hline Beserman $^{13}$ & - & - & 5.31 & - & - & - \\
\hline Hattori $^{14}$ & 9.92 & 6 & 5.40 & - & - & - \\
\hline Hidaka $^{15}$ & $7-10$ & - & - & - & - & - \\
\hline Peterson $^{16}$ & 10.1 & - & 5.43 & - & - & - \\
\hline $\operatorname{Ruda}^{17}$ & 9.67 & 7.28 & 6.27 & - & - & - \\
\hline $\mathrm{Oh}^{18}$ & - & - & 5.37 & - & - & - \\
\hline $\mathrm{Wu}^{19}$ & 10.17 & - & 5.30 & - & - & - \\
\hline Gallot $^{20}$ & 10.0 & - & 5.32 & $\leq 0.025$ & - & - \\
\hline Schall $^{21}$ & 9.62 & - & 5.32 & - & - & - \\
\hline Bignell $^{22}$ & 11.23 & 8.11 & 5.25 & 0.04 & - & - \\
\hline Mnasri $^{23}$ & - & 6.51 & 5.44 & - & - & - \\
\hline
\end{tabular}

compare with each other and the reviewed literature. By taking an average of the results for each wafer and using the upper and lower limits of their respective standard deviations as an uncertainty, we conclude that the low frequency dielectric constant is $\epsilon(0)=9.8 \pm 0.2$. When fitting this parameter, it was noticed that it was very stable relative to the other parameters, but depended strongly on the sample thickness. Each sample was measured separately to confirm its thickness. It was found that a change of $1 \%$ in $d$ could effect $\epsilon(0)$ by up to $5 \%$. The cross-correlation coefficients with respect to the rest of the parameters were generally less than 0.1 , and the parameter value scatter graphs were circular with crosssections of approximate Gaussian distribution.

Even though the measurements were not performed at high frequency, we were able to obtain reasonably accurate results for the high frequency dielectric constant. We attribute this to the fringe depth and width being dependent on this parameter. Our results fall within the spread of the previously published values and give an average value of $\epsilon(\infty)=7.3 \pm 0.6$ with the uncertainty representing the upper and lower limits of the standard deviation from each wafer. The large uncertainty can be attributed to a higher cross-correlation, particularly with the transverse optical phonon and plasma frequencies. Average cross-correlation coefficients for these pairs of parameters were around $0.6 \pm 0.2$. This is expected as each of these parameters contributes to separate terms in the dielectric function model. This was also confirmed by observing a slight linear dependence in the scatter graphs for each of these parameter pairs. Nevertheless, since the fitting algorithm was able to converge and the cross-correlation coefficients were much lower than 0.95 , we are confident that the results are accurate.

\section{Transverse optical phonon and phonon damping frequencies}

While the phonon frequency is an intrinsic property of the crystal, the damping constant will depend on the particular purity of the sample and is therefore not an intrinsic crystal property. Hence most of the reviewed literature only reports on the phonon frequency. Whilst the previously reported results agree within the uncertainty of the values presented in this article, the large deviation from the general consensus evident in Table I of $\nu_{\mathrm{TO}} \approx 5.3$ is notable. The accuracy does seem to increase with sample thickness. The uncertainty may be attributed to both the slight cross-correlation between the high frequency dielectric constants (described above) and the fact that the measurements have been taken in the sub- $\mathrm{THz}$ $(<1.0 \mathrm{THz})$ region. The sub-THz frequency region is an order of magnitude lower than the phonon resonance, and therefore we would expect much weaker coupling to the optical phonon. Many of the previous measurements ${ }^{10,13,22}$ were performed in reflection using far-infrared spectrometers which allows direct observation of the reststrahlen band. From this, the optical phonon frequencies can be directly determined with considerable accuracy as the actual structure of the resonance is observed. Taking an average of our results for each wafer gives $\nu_{\mathrm{TO}}=6.0 \pm 1.3 \mathrm{THz}$ with the uncertainty adjusted to encompass the full spread of the uncertainties for each wafer's results.

\section{Plasma and collision frequencies}

The plasma and collision frequencies represent the resonant frequency and damping of the charge carriers in the conduction band. As previously mentioned, these parameters are 
sample dependent and not intrinsic properties of the ZnTe crystal. This is why they do not appear in the reviewed literature. It could also explain the discrepancy between the results of the $1.0 \mathrm{~mm}$ sample compared to the others. While the 0.3 and $0.5 \mathrm{~mm}$ samples were obtained from one supplier the $1.0 \mathrm{~mm}$ sample was obtained from another and is likely to be of a different purity. The results of these parameters are, however, useful in determining the resistivity or carrier concentration of a particular sample. For example, using the results from the $0.5 \mathrm{~mm}$ sample and the form of the plasma frequency given earlier $\left(\omega_{\mathrm{p}}^{2}=n e^{2} / m^{*} \epsilon_{0} \epsilon(\infty)\right)$, we calculate an approximate carrier concentration of $n \approx 1.0 \times 10^{14} \mathrm{~cm}^{-3}$. This result agrees with concentrations for high resistivity $\mathrm{ZnTe}$ at room temperature. ${ }^{17} \mathrm{We}$ have used an effective electron mass of $m^{*}=0.151 m_{e}$ in this calculation. ${ }^{12}$

\section{CONCLUSIONS}

In summary, we have measured the transmittance of three $\mathrm{ZnTe}$ crystal wafers with different thicknesses using continuous-wave $\mathrm{THz}$ radiation. The transmittance spectra of these wafers allow the determination of the optical parameters of ZnTe by fitting the experimental data with theoretical curves calculated using a simple Drude-Lorentz dielectric function. The optical parameters include the low and high frequency dielectric constants and plasma and phonon frequencies as well as their damping constants. Due to the nonlinear dependence of the transmittance on these parameters, a statistical approach such as the bootstrap method should be used to obtain accurate values with genuine uncertainties. We show that our results fall within a broad range of acceptable values, which highlights the uncertainty in documented ZnTe optical parameters. Accurate knowledge of these parameters allows the calculation of carrier concentration and sample resistivity and demonstrates how a two-color photomixing $\mathrm{THz}$ source can perform as a semiconductor characterization tool. The excellent frequency precision, ability to perform contactless measurements, and low start up costs (particularly with their further developments to operate at telecommunication wavelengths) suggests that a two-color $\mathrm{THz}$ spectrometer is a good candidate for industry application.

\section{ACKNOWLEDGMENTS}

This work was supported by the Australian Research Council and by the University of Wollongong. We thank
Professor H. Hartnagel of the Technical University of Darmstadt for provision of the photomixer used in this work.

${ }^{1}$ K. Sakai, Terahertz Optoelectronics (Springer, New York, 2005).

${ }^{2}$ A. Nahata, A. S. Weling, and T. F. Heinz, Appl. Phys. Lett. 69, 2321 (1996).

${ }^{3}$ C. Winnewisser, P. U. Jepsen, M. Schall, V. Schyja, and H. Helm, Appl. Phys. Lett. 70, 3069 (1997).

${ }^{4}$ Z. G. Lu, P. Campbell, and X. C. Zhang, Appl. Phys. Lett. 71, 593 (1997).

${ }^{5}$ P. Y. Han and X. C. Zhang, Appl. Phys. Lett. 73, 3049 (1998).

${ }^{6}$ M. Aven and B. Segall, Phys. Rev. 130, 81 (1963).

${ }^{7}$ D. Berlincourt, H. Jaffe, and L. R. Shlozawa, Phys. Rev. 129, 1009 (1963).

${ }^{8}$ A. G. Fischer, J. N. Carides, and J. Dresner, Solid State Commun. 2, 157 (1964).

${ }^{9}$ D. T. F. Marple, J. Appl. Phys. 35, 539 (1964).

${ }^{10}$ A. Manabe, A. Mitsuishi, and H. Yoshinaga, Jpn. J. Appl. Phys. 6, 593 (1967).

${ }^{11}$ S. S. Mitra, O. Brafman, W. B. Daniels, and R. K. Crawford, Phys. Rev. 186, 942 (1969).

${ }^{12}$ D. L. Rode, Phys. Rev. B: Condens. Matter 2, 4036 (1970).

${ }^{13}$ R. Beserman and M. Balkanski, J. Phys. Chem. Solids 31, 355 (1970).

${ }^{14}$ T. Hattori, Y. Homma, and A. Mitsuishi, Opt. Commun. 7, 229 (1973).

${ }^{15}$ K. Hidaka and T. Kouno, J. Electrostat. 11, 195 (1982).

${ }^{16}$ D. L. Peterson, A. Petrou, W. Giriat, A. K. Ramdas, and S. Rodriguez, Phys. Rev. B: Condens. Matter 33, 1160 (1986).

${ }^{17}$ R. E. Ruda, J. Phys. D: Appl. Phys. 24, 1158 (1991).

${ }^{18}$ E. Oh, R. G. Alonso, I. Miotkowski, and A. K. Ramdas, Phys. Rev. B: Condens. Matter 45, 10934 (1992).

${ }^{19}$ Q. Wu and X. C. Zhang, IEEE J. Sel. Top. Quantum Electron. 2, 693 (1996).

${ }^{20}$ G. Gallot, J. Zhang, R. W. McGowan, T. I. Jeon, and D. Grischkowsky, Appl. Phys. Lett. 74, 3450 (1999).

${ }^{21}$ M. Schall, M. Walther, and P. U. Jepsen, Phys. Rev. B: Condens. Matter 64, 094301 (2001).

${ }^{22}$ L. J. Bignell and R. A. Lewis, J. Mater. Sci. Mater. Electron. 20, S326 (2009).

${ }^{23}$ S. Mnasri, S. A. Nasrallah, N. Sfina, N. Bouarissa, and M. Said, Semicond. Sci. Technol. 24, 095008 (2009).

${ }^{24}$ O. Morikawa, M. Tonouchi, and M. Hangyo, Appl. Phys. Lett. 75, 3772 (1999).

${ }^{25}$ J. Mangeney, F. Meng, D. Gacemi, E. Peytavit, J. F. Lampin, and T. Akalin, Appl. Phys. Lett. 97, 161109 (2010).

${ }^{26}$ E. D. Palik and J. K. Furdyna, Rep. Prog. Phys. 33, 1193 (1970).

${ }^{27}$ E. R. Brown, K. A. McIntosh, K. B. Nichols, and C. L. Dennis, Appl. Phys. Lett. 66, 285 (1995).

${ }^{28}$ D. Schoenherr, C. Bleasdale, T. Goebel, C. Sydlo, H. L. Hartnagel, R. Lewis, and P. Meissner, in IRMMW-THz 2010 - 35th International Conference on Infrared, Millimeter, and Terahertz Waves, 5-10 September 2010, Abstract No. 5613008.

${ }^{29}$ M. L. Johnson, Biophysical Tools for Biologists, Volume One: In Vitro Techniques, Methods in Cell Biology Vol. 84 (Academic, 2008), pp. 781805.

${ }^{30}$ M. L. Johnson, in Numerical Computer Methods, Part C, Methods in Enzymology Vol. 321 (Academic, 2000), pp. 424-446.

${ }^{31} \mathrm{~B}$. Efron and R. J. Tibshirani, An Introduction to the Bootstrap (Chapman and Hall, 1993). 